\title{
Late-onset, advanced scapular notching associated with a lateral offset reverse arthroplasty design - a
} case report

\begin{abstract}
Scapular notching is a recognized radiographic finding after reverse total shoulder replacement that has been associated with compromised patient outcomes. Notching has been less commonly described in implants with a center of rotation lateral to the glenoid. To the authors' knowledge, there have been no reports of notching greater than Sirveaux Grade 1 in lateralized implants. The current report describes the clinical course of a patient who developed progressive scapular notching after being treated with a DJO Encore reverse total shoulder prosthesis (Encore Medical, Austin, Texas), including intraoperative factors and findings and the progression of scapular notching through subsequent clinical visits. Her index surgery was performed for a massive rotator cuff tear with anterior superior escape and bone loss. Her initial radiographs and follow-up were unremarkable. At her 5-year follow-up, she had developed Grade 4 notching radiographically. The clinical significance of scapular notching has been debated in the literature. In this case, the patient's clinical outcome had not been compromised by the radiographic findings. Prosthesis orientation, such as anterior positioning of the baseplate resulting in posterior impingement of the implant, could also have led to radiographic notching. Although overall results with this prosthesis have been favorable, the authors recommend yearly radiographic follow-up to monitor for the development of notching. In this report, the authors also perform a brief review of current literature, including clinical results and implant design factors pertaining specifically to scapular notching of a lateralized offset reverse total shoulder prosthesis. Recognition of implant design and technical factors that contribute to scapular notching can help minimize this finding on follow-up of reverse total shoulder replacements.
\end{abstract}

Volume 6 Issue 4 - 2016

\author{
Steve A Petersen, ${ }^{1,2}$ Nickolas G Garbis ${ }^{1,2}$ \\ 'Department of Orthopaedic Surgery (NGG), Loyola University \\ Medical Center, USA \\ ${ }^{2}$ Department of Orthopaedics (SAP), The Johns Hopkins \\ University, USA
}

\begin{abstract}
Correspondence: Steve A Petersen, Department of Orthopedic Surgery, Medstar Good Samaritan Hospital, 560I Loch Raven Blvd, Suite G-I, Baltimore, USA, 21239, Tel 4434444740, Fax 4104444752 Email speter30@jhmi.edu
\end{abstract}

Received: September 27, 2016 | Published: November 2I, 2016

\section{Introduction}

Reverse shoulder arthroplasty, introduced by Grammont et al. ${ }^{1}$ in 1987 , is commonly associated with scapular notching, ${ }^{2}$ an impingement of the humeral cup against the scapula's inferior border. Although the clinical significance of notching has been debated, 2 studies reported less successful clinical outcomes in its presence., ${ }^{2,3} \mathrm{~A}$ laboratory study showed that a varus humeral neck-shaft angle and a lateral glenosphere offset reduce inferior scapular impingement. ${ }^{4}$ The DJO Encore reverse shoulder prosthesis (Encore Medical, Austin, Texas) has a varus, $135^{\circ}$ humeral neck-shaft angle and a lateralized offset glenosphere component. ${ }^{5}$ A literature search found 1 study. ${ }^{6}(5-$ year follow-up) and 1 case report.$^{7}$ describing notching associated with this reverse arthroplasty design, but neither reported more than a Sirveaux. ${ }^{2}$ Grade 1 notching.

The authors present a patient with Sirveaux Grade 4 scapular notching at 5 years after receiving a DJO Encore reverse total shoulder prosthesis and review the literature specific to scapular notching.

\section{Case report}

The patient presented as a 79-year-old right-hand-dominant woman with a 6-month history of atraumatic left shoulder pain, which she rated 6 on a 10-point scale. Elevation of the left arm measured $20^{\circ}$ actively and $110^{\circ}$ passively. Radiographs showed collapse of the anatomic humeral head with anterosuperior dislocation and loss of the glenohumeral joint space (Figure 1). She underwent a computed tomography scan of the shoulder to assess anterior bone loss. She consented to reverse shoulder arthroplasty, which was performed without complications. The implant used a 36-mm standard glenosphere with 6-mm lateral offset from the glenoid. The baseplate was positioned slightly more cephalad than the most inferior aspect of the glenoid to obtain optimal purchase of the locking screws. The early postoperative course was uneventful.
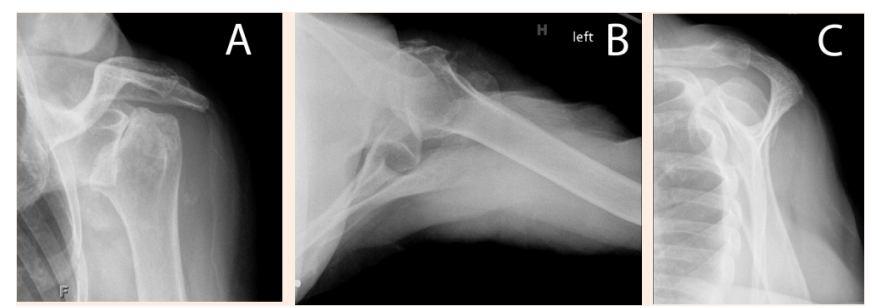

Figure I Grashey (A), axillary (B), and $Y(C)$ radiographs before surgery. There is collapse of the humeral head and anterosuperior subluxation.

Standard follow-up intervals were 2 and 6 weeks, 3 and 6 months, and 1,2 , and 5 years postoperatively. All radiographs were graded using the classification of Sirveaux et al. ${ }^{2}$

At 2-year follow-up, the patient rated her pain as 0 . Active forward elevation measured $60^{\circ}$. Radiographs obtained at that time showed Grade 1 notching (Figure 2). She continued to be satisfied with the result and to use a cane occasionally.

At 5-year follow-up, she continued to rate her pain as 0 and was satisfied. Lower-extremity osteoarthritis had progressed, and she used a walker for ambulation. Active elevation was $40^{\circ}$, and passive elevation was $130^{\circ}$. Radiographs obtained at that time showed Sirveaux Grade 4 notching involving the posterior inferior aspect of the glenoid (Figure 3). There was also a minimally displaced scapular spine fracture at the spinoglenoid notch. The patient was satisfied with her shoulder function. 

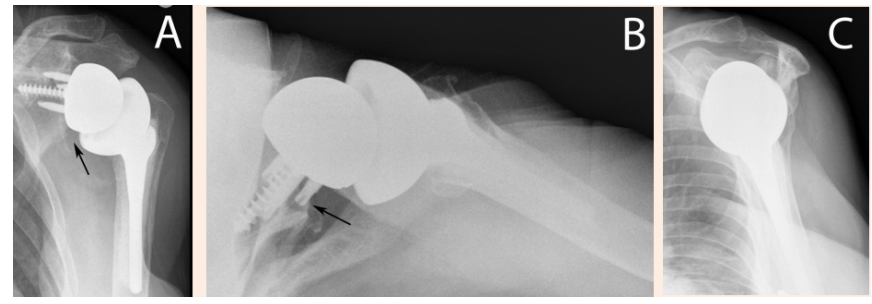

Figure 2 Grashey $(\mathrm{A})$, axillary $(\mathrm{B})$, and $\mathrm{Y}(\mathrm{C})$ radiographs 2 years after reverse total shoulder replacement with a DJO Encore prosthesis. There is an incomplete lucency involving the central screw and Grade 3 posterior-inferior notching best appreciated in the axillary radiograph. The arrows point to the areas of notching at the posterior-inferior glenoid.
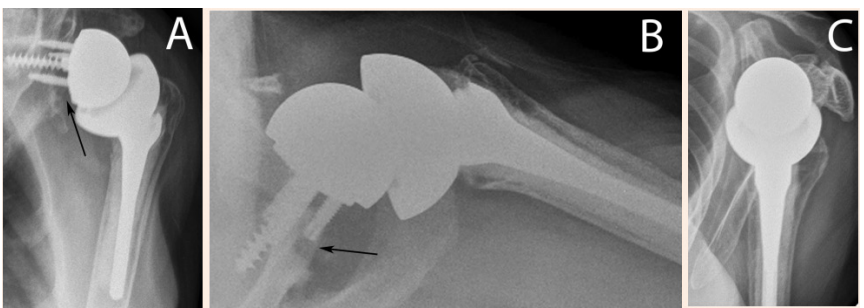

Figure 3 Grashey $(A)$, axillary $(B)$, and $Y(C)$ radiographs 5 years after reverse total shoulder replacement with a DJO Encore prosthesis. The patient had progression to Sirveaux Grade 4 scapular notching extending to the central screw. There is also an asymptomatic, minimally displaced scapular spine fracture. The arrows demonstrate progression of the posterior-inferior notching.

\section{Discussion}

Implant design has been shown to make a difference in the incidence of scapular notching in clinical and biomechanical studies. ${ }^{8,9}$ Current reverse total shoulder systems offer several different neck/ shaft angles, ranging from $135^{\circ}$ to $155^{\circ}$. Glenoid offset designs can range from 0 to $10 \mathrm{~mm}$ from the glenoid face. Gutierrez et al. ${ }^{8}$ performed a biomechanical study stratifying the effects of glenosphere diameter, center of rotation, glenosphere position on the glenoid, and humeral neck/shaft angle on inferior scapular impingement. Scapular impingement was most reduced by a varus humeral neck/shaft angle, followed by inferior glenosphere positioning on the glenoid and lateral glenosphere offset. Scapular impingement has also been shown to be reduced, but not eliminated, with an inferior offset or larger glenosphere in a Grammont-type prosthesis..$^{10,11}$

Cuff et al. ${ }^{5}$ published a minimum 5-year follow-up series of their lateral offset Encore reverse total shoulder arthroplasties and found that, of 76 patients, 7 (9\%) had notching, all of which was graded Sirveaux 1. In contrast, scapular notching has been a consistent radiographic finding in medial offset reverse arthroplasty designs despite efforts such as increasing inferior tilt or inferior placement of the glenosphere to limit its occurrence. ${ }^{11}$ Boileau et al. ${ }^{12}$ used a 7-mm or 10-mm autologous disk of cancellous bone graft beneath an extended central peg baseplate and glenosphere, lateralizing the glenoid but maintaining the center of rotation at the glenosphere-graft interface to reduce stress at that interface. Despite this effort to reduce scapular notching, 19\% ( 8 of 42) of their patients had notching at a mean follow-up of 28 months (range, 24-40). Progression of scapular notching has also been reported for medial offset designs. Levigne et al. ${ }^{12}$ noted Sirveaux Grade 1 scapular notching in $48 \%$ of 461 medial offset reverse total shoulders at 1 year, $60 \%$ at 2 years, and $68 \%$ at 3 years. Notching was usually apparent early in follow-up in this series. Valenti et al. ${ }^{11}$ reported on "less medialized" glenospheres having decreased notch rates, with improved rotation.
Grading of scapular notching is influenced by the baseplate screw design particular to the reverse designs available. The DJO Encore system has a 4-hole baseplate with a fixed central screw and a protocol that implants the baseplate in $10^{\circ}$ to $15^{\circ}$ of inferior tilt. ${ }^{13}$ This construct allows the inferior screw to be slightly superiorly directed, in contrast to many of the medial offset reverse baseplate designs that rely on an inferiorly directed screw for secure bone purchase. Given that the Sirveaux classification is based on the relation of bone loss to the inferior screw, the degree of scapular notching for the Encore prosthesis may not be as appreciated as other designs. As noted in the literature and with this patient's follow-up, continued radiographic follow-up of a reverse shoulder arthroplasty, regardless of design, is warranted for the surveillance of scapular notching.

With regard to this patient, there were technical components that contributed to the development of notching. The baseplate was not at the most inferior position of the glenoid, which can predispose to notching regardless of implant design. Superior placement of the glenoid baseplate was influenced by anterior glenoid bone loss that was not augmented with bone graft. The baseplate was subsequently positioned to optimize the purchase of the central and peripheral locking screws.

The postoperative radiographs had slightly different projections from year to year that interfered with the consistent location and grading of scapular notching. Pre-existing anterior glenoid bone loss could be mistaken for anterior inferior scapular notching that was, in fact, not present.

\section{Conclusion}

Although the rate of notching using lateral offset reverse total shoulder prosthesis is low, scapular notching may progress postoperatively in some patients. Despite having prosthetic design features that avoid scapular notching, the authors have identified progressive, advanced scapular notching in a lateral offset reverse shoulder replacement. A false security may exist that notching is prevented with lateral offset designs despite baseplate position. For this patient, the glenoid baseplate was positioned to optimize screw purchase, a situation that will likely challenge other surgeons confronted with glenoid bone deficiency or poor bone quality.

Given the authors' experience, it is their hope to raise awareness among surgeons who prefer lateralized reverse arthroplasty design features about the possibility of progressive scapular notching and a need for continued patient follow-up. The degree of scapular notching may also be more difficult to assess in prosthetic designs with baseplates that have superiorly directed screws, as in the DJO Encore design. The authors advise radiographic follow-up of this reverse shoulder implant design beyond 2 years and recommend closer follow-up for patients who use assistive walking devices or develop the onset of shoulder pain.

\section{Acknowledgments}

None.

\section{Conflicts of interest}

None.

\section{References}

1. Gouider-Khouja N, Kraoua I, Benrhouma H et al. Movement disorders in neuro-metabolic diseases. Eur J Paediatr Neurol. 2010;14(4):304-307.

2. Grammont P, Trouilloud P, Laffay JP Etude et realisation d'une nouvelle prothese d'epaule. Rhumatologie. 1987;39(10):407-418. 
3. Sirveaux F, Favard L, Oudet D et al. Grammont inverted total shoulder arthroplasty in the treatment of glenohumeral osteoarthritis with massive rupture of the cuff. Results of a multicentre study of 80 shoulders. J Bone Joint Surg Br. 2004;86(3):388-395.

4. Sadoghi P, Leithner A, Vavken P et al. Infraglenoidal scapular notching in reverse total shoulder replacement: a prospective series of 60 cases and systematic review of the literature. BMC Musculoskelet Disord. 2011;2:101.

5. Gutierrez S, Levy JC, Lee WE et al. Center of rotation affects abduction range of motion of reverse shoulder arthroplasty. Clin Orthop Relat Res. 2007;458:78-82.

6. Frankle M, Siegal S, Pupello D et al. The Reverse Shoulder Prosthesis for glenohumeral arthritis associated with severe rotator cuff deficiency. A minimum two-year follow-up study of sixty patients. J Bone Joint Surg Am. 2005;87(8):1697-1705.

7. Cuff D, Clark R, Pupello D et al. Reverse shoulder arthroplasty for the treatment of rotator cuff deficiency: a concise follow-up, at a minimum of five years, of a previous report. J Bone Joint Surg Am. 2012;94(21):1996-2000.

8. Levy J, Blum S Inferior scapular notching following encore reverse shoulder prosthesis. Orthopedics. 2009;32(10).
9. Gutiérrez S, Levy JC, Frankle MA et al. Evaluation of abduction range of motion and avoidance of inferior scapular impingement in a reverse shoulder model. J Shoulder Elbow Surg. 2008;17(4):608-615.

10. Kempton LB, Balasubramaniam M, Ankerson E et al. A radiographic analysis of the effects of prosthesis design on scapular notching following reverse total shoulder arthroplasty. J Shoulder Elbow Surg. 2011;20(4):571-576.

11. Boughebri O, Duparc F, Adam JM, Valenti P Arthroscopic dynamic analysis of scapular notching in reverse shoulder arthroplasty. Orthop Traumatol Surg Res. 2011;97(8):779-784.

12. Valenti P, Sauzieres P, Katz D et al. Do less medialized reverse shoulder prostheses increase motion and reduce notching? Clin Orthop Relat Res. 2011;469(9):2550-2557.

13. Levigne C, Garret J, Boileau $\mathrm{P}$ et al. Scapular notching in reverse shoulder arthroplasty: is it important to avoid it and how? Clin Orthop Relat Res. 2011;469(9):2512-2520.

14. Gutierrez S, Greiwe RM, Frankle MA et al. Biomechanical comparison of component position and hardware failure in the reverse shoulder prosthesis. J Shoulder Elbow Surg. 2007;16(3 Suppl):9S-12S. 